\title{
Lamotrigine-Valproic Acid combination leading to severe skin drug reactions in children
}

\author{
Radia Chakiri ${ }^{1}$, Khalila Nainia ${ }^{1}$, and Youssef Khabbal $^{1}$ \\ ${ }^{1}$ Université Ibn Zohr
}

March 6, 2022

\begin{abstract}
Drug exposure is the most common cause of severe cutaneous reactions. Toxic epidermal necrolysis, erythroderma, drug rash with eosinophilia and systemic symptoms, acute generalized exanthematous pustulosis and drug induced vasculitis are the common cutaneous drug reactions which can have severe morbidity and evenmortality. Antiepileptics are among the drugs incriminated.
\end{abstract}

\section{Introduction}

Lamotrigine (LTG) is an important anticonvulsant with activity against both focal and generalized onset seizures. [1] However, its use is associated with a significant incidence of adverse cutaneous reactions, mainly if it is used in combination with other therapy. [2] Rash is the most common adverse reaction of this antiepileptic agent and the most common reason for treatment discontinuation as it can be life-threatening. Children under polytherapy have a higher risk of this adverse event. Such cutaneous reactions range from mild rashes to more serious conditions. [3]

Here, we present two cases of severe skin drug reactions that occurred after addition of LTG to the Valproic acid regimen.

\section{Case 1}

A 9-year-old girl attended to our hospital with a generalized itchy skin eruption starting from the face, trunk and extremities for four days. She was diagnosed with epilepsy and treated with Valporat-acid for 3 years. Lamotrigine $(25 \mathrm{mg} /$ day) was added to her regimen 2 weeks before the eruptions due to uncontrolled seizures. On examination she was alert and oriented with a temperature of $39^{\circ} \mathrm{C}$ and pulse was $112 /$ minutes. There was a generalized erythematous maculopapular skin eruptions that were localized in the trunk, extremities and face, erythematous target and purpuric lesions in the lower limbs, edema of the face and swollen lips. [Figure 1a; 1b]The examination of the rest of the body was normal, especially the lymph node areas were free.

The laboratory investigation showed that C-reactive protein: 100mg/dl, WBC: $9750 / \mathrm{mm}^{3}$, Neutrophil: $81 \%$ dominant $\left(7910 / \mathrm{mm}^{3}\right.$ ), Lymphopenia at $740 / \mathrm{mm}^{3}$, Na: $128 \mathrm{mEq} / \mathrm{L}$ (Normal range: $135-145 \mathrm{mEq} / \mathrm{L}$ ). other electrolytes, liver and kidney functions were within normal limit. The skin pathology of a target lesion was consistent with erythema multiforme. Declaration to pharmacovigilance confirmed the imputability of the combination of Lamotrigine with Valporat Acid in the occurrence of drug-induced skin reaction.

The final diagnosis was DRESS syndrome based on the presence of fever, face oedema, occurrence of the itchy polymorphic generalized skin eruption 2 weeks after the onset of the lamotrigine and the presence of lymphopenia. 
Lamotrigine and Valporat Acid were immediately discontinued and the patient was treated with prednisone at a dose of $0,5 \mathrm{mg} / \mathrm{kg} /$ day with good outcomes.

\section{Case 2}

A 5-year-old girl attended to our hospital for generalized skin eruption starting from abdominal region, oral mucosa erosion the extension to the rest of the body. She was diagnosed with epilepsy and treated with Valporat Acid for 8 months. Lamotrigine was added to her regimen 20 days before the eruption due to uncontrolled seizures. No other medication or recent infection was present in the past medical history. Dermatological examination revealed: oral mucosal ulcerations, hemorrhagic crust on lips and nose, erythematous papules and bullae located mainly on the face, neck, trunk and upper and lower extremities. There were confluent bullae formation and epidermal loss located especially on his face, back and abdomen that accounted for approximately $30 \%$ of the total body surface area. [Figures 2a; 2b]

Laboratory examinations, including complete blood count, liver and renal function tests, electrolytes, urine analysis were within normal limit. The skin pathology of cutaneous specimen revealed a subepidermal blisters with widespread necrosis and apoptotic keratinocytes associated with minimal lymphocytic inflammatory infiltrate. Thus the diagnosis of Lyell syndrome was made. The declaration to pharmacovigilance confirmed the imputability of the combination of Lamotrigine with Valporat Acid in the occurrence of Lyell syndrome.

Lamotrigine and Valporat Acid were immediately discontinued and the patient was treated symptomatically with good outcomes.

\section{Discussion}

In pediatric population, cutaneous reactions constitute $35 \%$ to $36 \%$ of adverse drug reactions. [4] Despite the high prevalence of cutaneous adverse drug reactions, they are mostly of benign character and cause mild clinical symptoms and subside spontaneously with discontinuation of the suspected drug. [5] However, $2 \%$ to $6.7 \%$ Cutaneous adverse drug reactions can be severe and potentially life-threatening clinical syndromes. $[6 ; 7]$

Drug reaction with eosinophilia and Systemic Symptoms (DRESS), Steven-Johnson syndrome (SJS), and toxic epidermal necrolysis (TEN) are among the severe cutaneous adverse drug reactions as in our two cases.

DRESS syndrome is a rare, potentially life-threatening delayed drug induced hypersensitivity reaction. Symptoms include skin eruptions and systemic symptoms of fever, malaise, and lymphadenopathy. Additional symptoms are related to visceral involvement such as altered liver function, nephritis, and pneumonitis. Hematologic abnormalities may include leukocytosis with eosinophilia, thrombocytopenia, and atypical lymphocytosis. [8; 9] The syndrome begins within 2 to 6 weeks after drug introduction as in our first case. Reactivation of herpesviruses has also been shown to play a role in the pathogenesis of DRESS syndrome, especially human herpesvirus-6 (HHV-6) but also cytomegalovirus, Epstein-Barr virus (EBV). [10]

SJS and TEN are diseases within the spectrum of severe cutaneous adverse reactions affecting skin and mucous membranes. [11] Initial symptoms of both TEN and SJS can be fever, stinging, and pain upon swallowing, any of which can precede cutaneous manifestations by 1 to 3 days. Skin lesions tend to first appear on the trunk, spreading to the neck, face, and proximal upper extremities. The distal portions of the arms as well as legs are relatively spared, but the palms and soles can be an early site of involvement. Erythema and erosions of the buccal, ocular, and genital mucosa are present in more than $90 \%$ of patients. The epithelium of the respiratory tract is involved in $25 \%$ of cases of TEN, and gastrointestinal lesions can also occur. The skin lesions are usually tender, and mucosal erosions are very painful. $[12,13]$ The total body surface area (TBSA) of the detachment is the main distinguishing factor between SJS, SJS-TEN overlap and TEN. SJS presents with epidermal detachment of less than 10\% TBSA, whereas involvement of 10-30\% TBSA is defined as an SJS/TEN overlap. TEN is defined as epidermal detachment of $30 \%$ TBSA or more. Epidermal loss in our second case accounted for approximately 10\% TBSA, so our diagnosis was SJS. [11]

Antiepileptic drugs are implicated in the occurrence of severe cutaneous adverse reactions, 
Several antiepileptic drugs are used in combination when seizures are poorly controlled. This fact leads to more potential pharmacokinetic and pharmacodynamic interactions in comparison with monotherapy. [14]

When LTG was started, the patients were also receiving VPA for several years for the first case and several months for the second, and no cutaneous adverse effects were recorded during this therapy. However, VPA even at low concentrations is known to decrease LTG clearance leading to higher serum concentrations of LTG which in turn increases the risk of serious rash. [15, 16, 17]

VPA is mainly metabolized by the liver, and it is a known inhibitor of UDP-glucuronosyltransferases; [15] according to some investigations, [16]

there was a significant increase in LTG serum concentrations from $4.67 \pm 3.66$ to $9.56 \pm 5.27 \mu \mathrm{g} / \mathrm{mL}$ by concomitant administration of VPA, indicating a decrease in LTG clearance when VPA was added to therapy. In this case, the minor elimination pathway of LTG would play a more important role, and the formation of the arene oxide metabolite would be enhanced. As this epoxide appears to be detoxified mainly by enzymatic reactions, involving microsomal epoxide hydrolase (EPHX) and/or GSH-S-transferases and these enzymes are polymorphologically expressed in humans, [18] arene oxide toxicity is increased when EPHX or GSH S-transferases is either defective or inhibited or a depletion of intracellular glutathione levels is taking place. Inhibition of EPHX can occur when VPA is coadministred. [19, 20] High concentrations of VPA can deplete glutathione levels. [21] These two facts could lead to the frequent adverse effects. [22, 23]

Considering serious morbidity with mortality, early treatment is crucial for severe skin drug reactions. Thus, in all cases, the mean treatments are the withdrawal of the culprit drug and management of symptoms. [24] Specific treatment in children is controversial. For the treatment of SJS/TEN, systemic steroids, IVIG appeared to have better outcomes. [4] However in DRESS syndrome the main treatment is the administration of systemic steroid, other treatment modalities such us IVIG, plasmapheresis, cyclophosphamide, and cyclosporine have been also used. [24]

\section{Conclusion}

In these cases, we would like to emphasize that the combination of lamotrigine and valproic acid to control seizures can cause life-threatening skin reactions such as SJS/TEN and DRESS syndrome. the physicians should be alert and have to be aware of that when prescribing antiepileptic drugs.

\section{References}

1. A.WArshavsky, A.Eilam, and R.Gilad. Lamotrigene as a monotherapy in clinical practice: efficacy of various dosages in epilepsy. Brain and Behaviour. Vol6. N³. Article e00419.

2. J. R. Calabrese, J. R. Sullivan, C. L. Bowden et al., "Rash in multicenter trials of lamotrigine in mood disorders: clinical relevance and management," Journal of Clinical Psychiatry, vol. 63, no. 11, pp. 1012-1019, 2002.

3. O. Egunsola, I. Choonara, and H. M. Sammons, "Safety of lamotrigine in paediatrics: a systematic review," BMJ Open, vol. 5, no. 6, article e007711, 2015.

4. Noguera-Morel L, Hernandez-Martin A, Torrelo A. Cutaneous drug reactions in the pediatric population. Pediatr Clin North Am 2014; 61: 403-26.

5. Gomes E, Brockow K, Kuyucu S, Saretta F, Mori F, Blanca-Lopez N, et al. Drug hypersentivity in children : report from the pediatric Task Force of the EAACI Drug Allergy Interest Group. Allergy 2016; 71: 149-61.

6. Teo YX, Walsh SA. Severe drug reactions. Clin Med 2016; 16: 79-83.

7. Hoetzenecker. W, Nägeli MM, Mehra ET, Jensen AN, Saulite I, Schimid-Grendelmeier P, et al. Adverse cutaneous drug eruptions : current understanding. Semin Immunopathol $2016 ; 38: 75-86$.

8. Husain Z, Reddy BY, Schwartz RA. DRESS syndrome: Part I. Clinical perspectives. J Am Acad Dermatol 2013;68:693.e1-693.e14. quiz 706-708.

9. Cacoub P, Musette P, Descamps V, Meyer O, Speirs C, Finzi L, et al. The DRESS syndrome: a literature review. Am J Med 2011;124:588-97. 
10. Marcus N, Smuel K, Almog M, Prais D, Straussberg R, Landau D, et al. Successful Intravenous Immunoglobulin Treatment in Pediatric Severe DRESS Syndrome. J Allergy Clin Immunol Pract. août 2018;6(4):1238-42.

11. Yapici AK, Fidanci MK, Kilic S, Balamtekin N, Mutluay Arslan M, Yavuz ST, et al. Stevens-Johnson Syndrome triggered by a combination of clobazam, lamotrigine and valproic acid in a 7-year-old child. Ann Burns Fire Disasters. 30 sept 2014;27(3):121-5.

12. Mockenhaupt M, Viboud C, Dunant A et al.: Stevens-Johnson syndrome and toxic epidermal necrolysis: assessment of medication risks with emphasis on recently marketed drugs. The EuroSCARstudy. J Invest Dermatol, 128: 35-44, 2008.

13. French LE: Toxic epidermal necrolysis and Stevens Johnson syndrome: our current understanding. Allergol Int, 55: 9-16, 2006.

14. Lamotrigine-Valproic Acid Interaction Leading to Stevens-Johnson Syndrome - PubMed [Internet]. [cite 25 fevr 2021]. Disponible sur:https://pubmed.ncbi.nlm.nih.gov/30228819/

15. W. Lu and J. P. Uetrecht, "Possible bioactivation pathways of lamotrigine," Drug Metabolism and Disposition, vol. 35, no. 7, pp. 1050-1056, 2007.

16. M. Lalic, J. Cvejic, J. Popovic et al., "Lamotrigine and valproate pharmacokinetics interactions in epileptic patients,"European Journal of Drug Metabolism and Pharmacokinetics, vol. 34, no. 2, pp. 93-99, 2009

17. A. Rowland, D. J. Elliot, J. A. Williams, P. I. Mackenzie, R. G. Dickinson, and J. O. Miners, "In vitro characterization of lamotrigine N2-glucuronidation and the lamotrigine-valproic acid interaction," Drug Metabolism and Disposition, vol. 34, no. 6, pp. 1055-1062, 2006.

18. L. W. Wormhoudt, J. N. Commandeur, and N. P. Vermeulen, "Genetic polymorphisms of human $\mathrm{N}$-acetyltransferase, cytochrome $\mathrm{P} 450$, glutathione S-transferase, and epoxide hydrolase enzymes: relevance to xenobiotic metabolism and toxicity," Critical Reviews in Toxicology, vol. 29, no. 1, pp. 59-124, 1999.

19. O. Spiegelstein, D. L. Kroetz, R. H. Levy et al., "Structure activity relationship of human microsomal epoxide hydrolase inhibition by amide and acid analogues of valproic acid," Pharmaceutical Research, vol. 17, no. 2, pp. 216-221, 2000.

20. M. Rosa, P. Bonnaillie, and H. Chanteux, "Prediction of drug-drug interactions with carbamazepine10,11-epoxide using a new in vitro assay for epoxide hydrolase inhibition," Xenobiotica, vol. 46, no. 12, pp. 1076-1084, 2016.

21. D. Diki'c, D. Jutri'c, and K. Dominko, ")e dual nature of the antiepileptic drug valproic acid, with possible beneficial effects in Alzheimer's disease," SEEMEDJ, vol. 1, no. 1, pp. 74-89, 2017.

22. X. Wang, B. Iv, H. Wang et al., "Lamotrigine-induced severe cutaneous adverse reaction: update data from 1999-2014," Journal of Clinical Neuroscience, vol. 22, no. 6, pp. 1005-1011, 2015.

23. K. Maduemem, A. Vatca, T. O'Neill, and D. Buckley, "Stevens- Johnson syndrome induced by combination of lamotrigine and valproic acid in a 9-year-old boy," Irish Medical Journal, vol. 110, no. 6, p. 586, 2017.

24. Dibek Misirlioglu E, Guvenir H, Bahceci S, Haktanir Abul M, Can D, Usta Guc BE, et al. Severe Cutaneous Adverse Drug Reactions in Pediatric Patients: A Multicenter Study. J Allergy Clin Immunol Pract. juin 2017;5(3):757-63. 


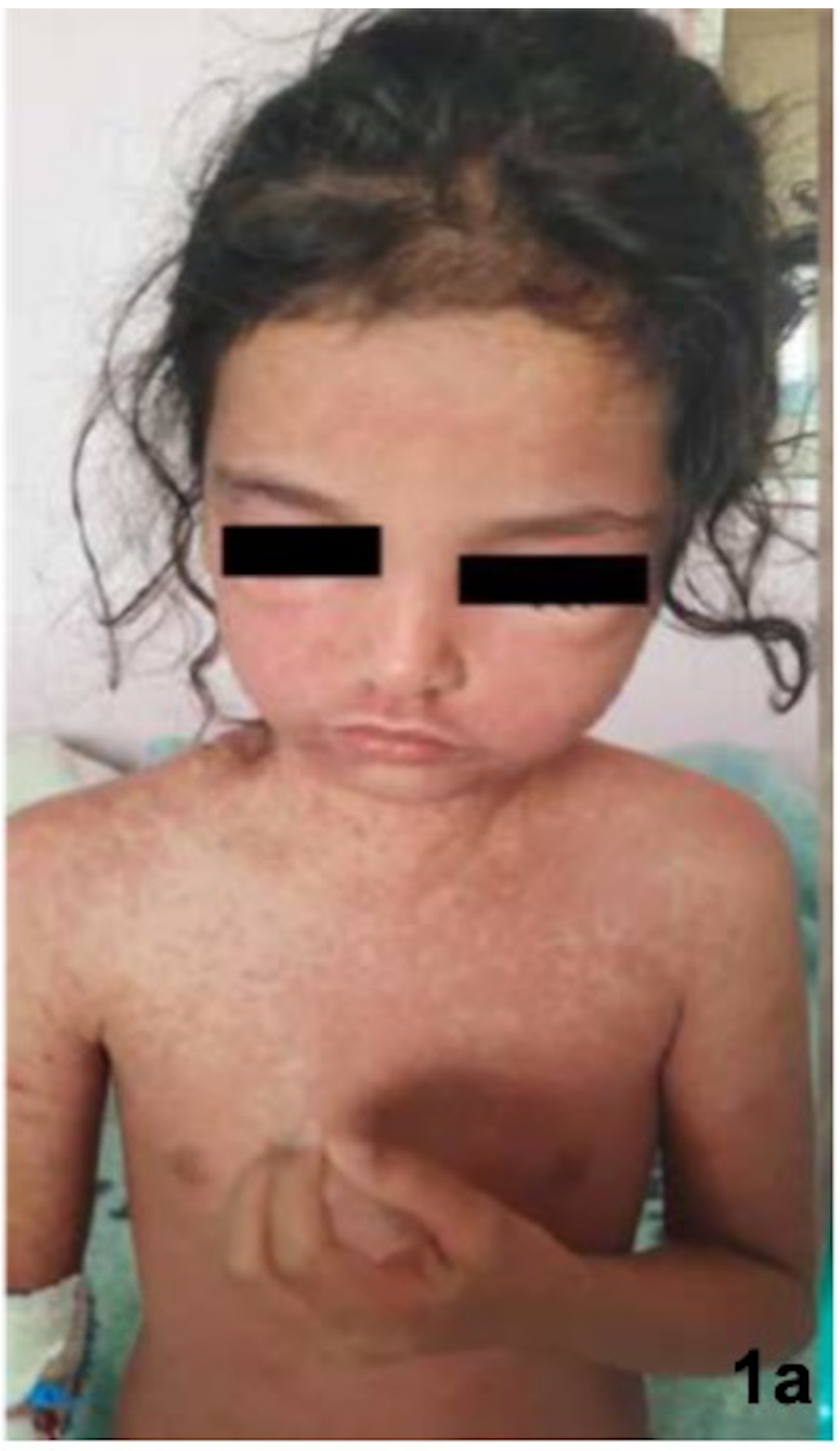




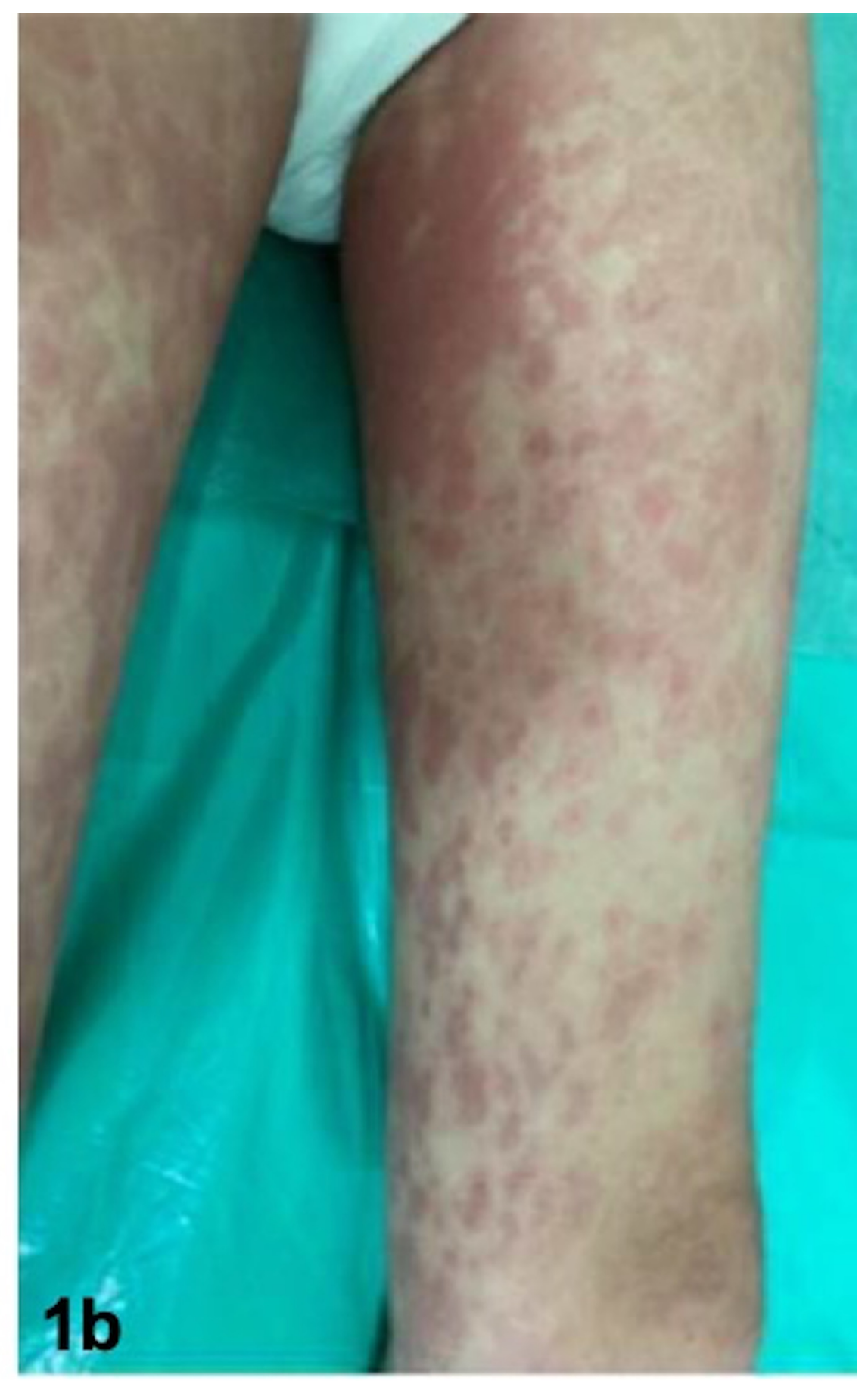




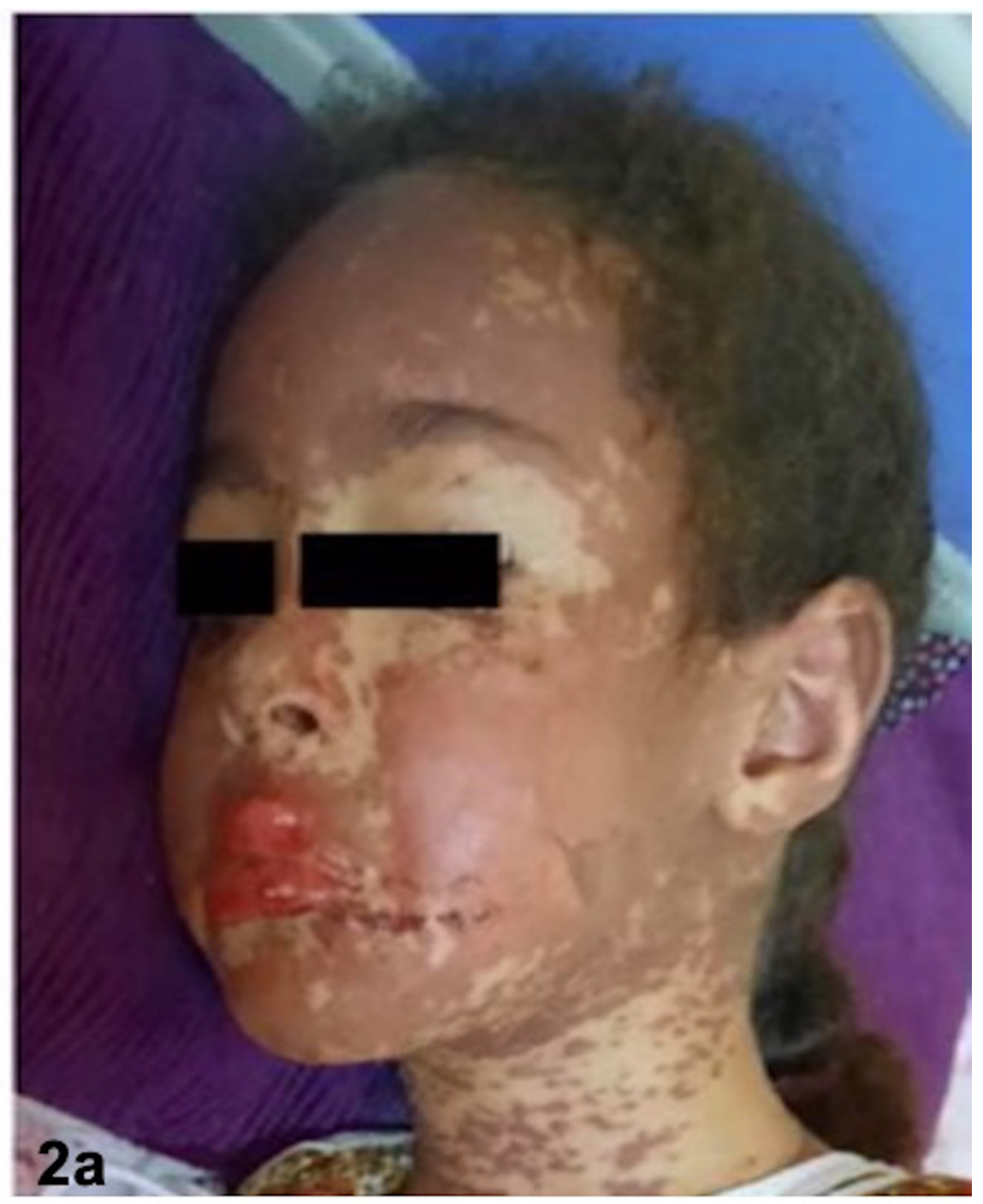




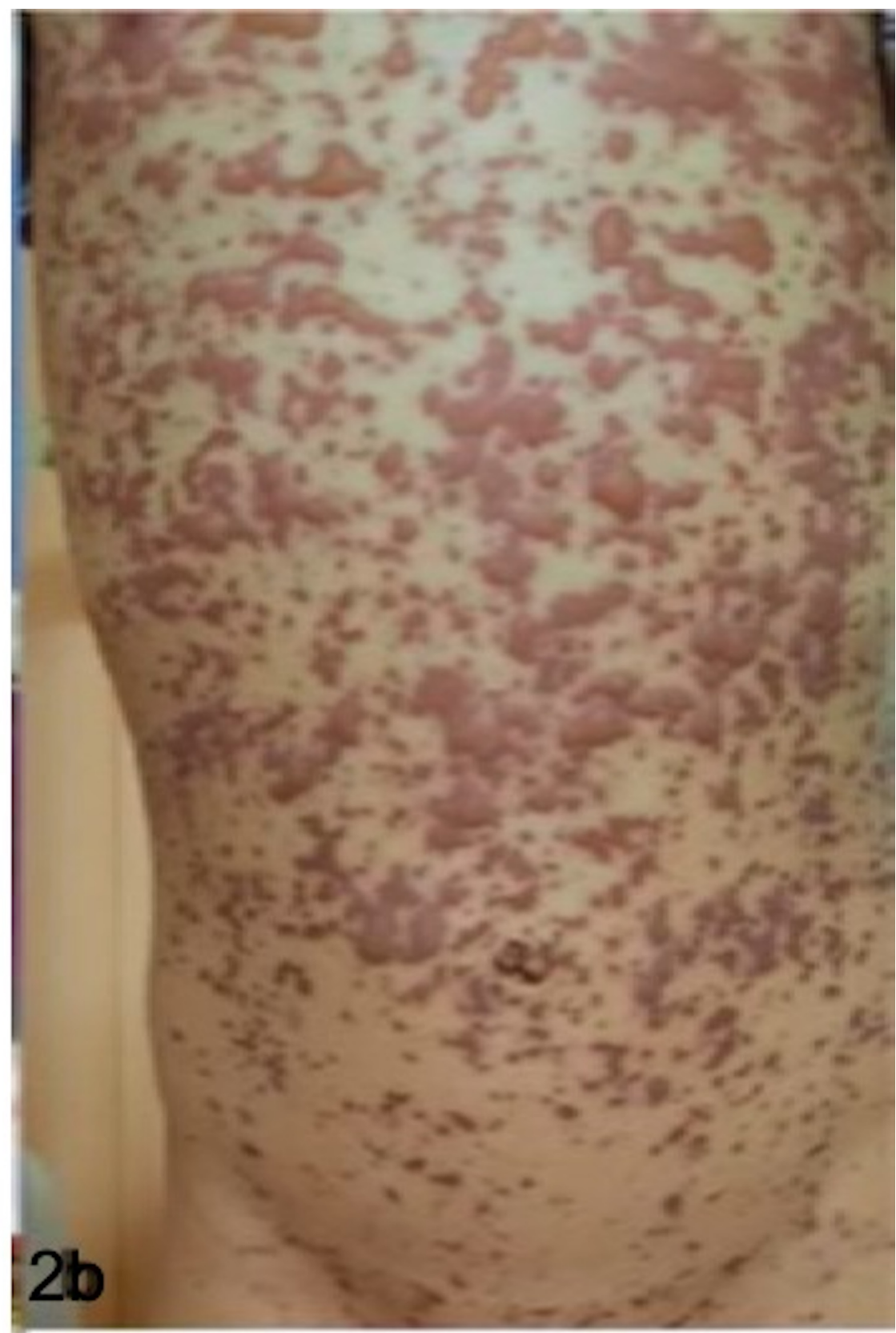

